This item was submitted to Loughborough's Research Repository by the author.

Items in Figshare are protected by copyright, with all rights reserved, unless otherwise indicated.

\title{
Reduction to modified KdV and its KP-like generalization via phase modulation
}

\section{PLEASE CITE THE PUBLISHED VERSION}

https://doi.org/10.1088/1361-6544/aabfab

\section{PUBLISHER}

(c) IOP Publishing Ltd \& London Mathematical Society

\section{VERSION}

AM (Accepted Manuscript)

\section{PUBLISHER STATEMENT}

This work is made available according to the conditions of the Creative Commons Attribution-NonCommercialNoDerivatives 3.0 Unported (CC BY-NC-ND 3.0) licence. Full details of this licence are available at: http://creativecommons.org/licenses/by-nc-nd/3.0/

\section{LICENCE}

CC BY-NC-ND 3.0

\section{REPOSITORY RECORD}

Ratliff, Daniel J., and Thomas J. Bridges. 2019. "Reduction to Modified Kdv and Its Kp-like Generalization via Phase Modulation”. figshare. https://hdl.handle.net/2134/35899. 


\title{
Reduction to modified KdV and its KP-like generalization via phase modulation
}

\author{
Daniel J. Ratliff \& Thomas J. Bridges \\ Department of Mathematics, University of Surrey, Guildford GU2 7XH, UK \\ E-mail: D.Ratliff@surrey.ac.uk
}

8th November 2018

\begin{abstract}
The main observation of this paper it that the modified Korteweg-de Vries equation has its natural origin in phase modulation of a basic state such as a periodic travelling wave or more generally a family of relative equilibria. Extension to $2+1$ suggests that a modified Kadomtsev-Petviashvili (or a Konopelchenko-Dubrovsky) equation should emerge, but our result shows that there is an additional term which has gone heretofore unnoticed. Thus through the novel application of phase modulation a new equation appears as the $2+1$ extension to a previously known one. To demonstrate the theory it is applied to the cubic-quintic Nonlinear Schrödinger (CQNLS) equation, showing that there are relevant parameter values where a modified KP equation bifurcates from periodic travelling wave solutions of the $2+1$ CQNLS equation.
\end{abstract}

PACS numbers: 47.10.Df, 47.35.-i, 42.10, 45.20.Jj

Keywords: nonlinear waves, Lagrangian fields, phase dynamics

Submitted to: Nonlinearity

\section{Introduction}

The modified Korteweg-de Vries equation $(\mathrm{mKdV})$,

$$
u_{t} \pm u^{2} u_{x}+u_{x x x}=0
$$

for the scalar-valued function $u(x, t)$ is most well-known as an integrable member of the KdV family. The interest of this paper will be on how the mKdV (and its $2+1$ generalisations) emerge in a reduction from an arbitrary PDE generated by a Lagrangian. The properties and solutions of the mKdV equation motivate this analysis, but are not the focus of this paper. 
The mKdV equation is related to the KdV equation through the Miura transformation [12]. However, since the Miura transformation is not bijective, the range of solutions of mKdV is much larger than $\mathrm{KdV}[20]$. For example, the mKdV equation has breather solutions and the cnoidal waves are Benjamin-Feir unstable when (1.1) has the plus sign [7, 20]. The $\mathrm{mKdV}$ has been found in a wide range of applications: Alfven waves, anharmonic lattices, ion acoustic solitons, traffic flow, thin ocean jets, heat pulses in solids, and internal waves (see [20] and references therein).

Typically the $\mathrm{KdV}$ or $\mathrm{mKdV}$ is derived starting with the dispersion relation in the neighborhood of a trivial state. The dispersion relation generates the linear terms and an asymptotic expansion or the method of multiples scales is used to generate the nonlinear terms (e.g. [6]). In this paper this strategy is reversed; the basic state is used as the generator of the mKdV equation. This strategy is implemented using phase modulation. It is natural since it encapsulates the case of emergence from simple or trivial basic states but also generalises to the case of bifurcation from periodic travelling waves. Phase modulation dramatically enlarges the range of contexts where the mKdV emerges as a model.

Phase modulation has its origins in Whitham modulation theory (WMT) [19] but WMT generates dispersionless modulation equations. The idea in phase modulation is to use a more general scaling and a direct ansatz with explicit wavenumber and frequency modulation (e.g. $[1,16,17,18])$, taking into account natural singularities. With the right scaling dispersion enters naturally.

As in WMT, the starting point is the class of PDEs that are the Euler-Lagrange equation associated with a general Lagrangian density,

$$
\mathscr{L}(U)=\iiint \mathcal{L}\left(U, U_{t}, U_{x}, U_{y}\right) \mathrm{d} x \mathrm{~d} y \mathrm{~d} t
$$

for some vector-valued function $U(x, y, t)$. The theory will be developed in the context of $2+1$ and this will lead to more than the mKdV equation and indeed more than the modified KP equation. The theory revolves around the basic state. For definiteness the basic state is assumed to be a periodic travelling wave solution

$$
U(x, y, t)=\widehat{U}\left(k x+m y+\omega t+\theta_{0}\right)=\widehat{U}(\theta), \quad \widehat{U}(\theta+2 \pi)=\widehat{U}(\theta),
$$

where $(k, m)$ are the wavenumbers and $\omega$ is the frequency, although the theory can be built around any relative equilibrium (cf. $[1,16,17,18]$ ). The Lagrangian, averaged over one period, on the wavetrain is then

$$
\mathscr{L}(\omega, k, m)=\int_{0}^{2 \pi} \mathcal{L}\left(\widehat{U}, \omega \widehat{U}_{\theta}, k \widehat{U}_{\theta}, m \widehat{U}_{\theta}\right) \mathrm{d} \theta .
$$

The Whitham strategy is to substitute $k=\theta_{x}, m=\theta_{y}, \omega=\theta_{t}$, and take the variation of (1.4) with respect to $\theta$ which results in the conservation of wave action,

$$
\mathscr{A}(\omega, k, m)_{t}+\mathscr{B}(\omega, k, m)_{x}+\mathscr{C}(\omega, k, m)_{y}=0,
$$


where $\omega, k$, and $m$ are considered as functions of $x, y, t$, and $\mathscr{A}:=\mathscr{L}_{\omega}, \mathscr{B}:=\mathscr{L}_{k}$, and $\mathscr{C}:=\mathscr{L}_{m}$. This equation, coupled with the consistency conditions,

$$
k_{t}=\omega_{x}, \quad m_{t}=\omega_{y}, \quad k_{y}=m_{x}
$$

form the Whitham modulation equations (WMEs). However the four equations (1.5)-(1.6) are a set of dispersionless first-order PDEs. To generate the $\mathrm{mKdV}$ equation a more general approach is needed.

Our strategy here is to use an explicit ansatz for the modulation. That is the modulation of $\widehat{U}(\theta, \omega, k, m)$ is represented by

$$
\widehat{U}\left(\theta+\varepsilon^{a} \phi, \omega+\varepsilon^{b} \Omega, k+\varepsilon^{c} q, m+\varepsilon^{d} r\right)+\varepsilon^{e} W,
$$

where $W$ is a remainder, $\varepsilon$ is a small parameter, the exponents $a, b, c, d, e$ are problem dependent, and $\Omega, q$, and $r$ depend on slow time and space scales. Singularities in wavenumber-frequency space affect the choice of scales, and are the key mechanism for bringing in dispersion. Substitution of (1.7) into the Euler-Lagrange equation, expansion, and reduction then leads to PDEs for $\Omega, q$ and $r$. The aim is to identify the singularites and scaling that result in a $\mathrm{mKdV}$ in $1+1$ and its generalisation to $2+1$.

The main result of this paper is that when the components $(\mathscr{A}, \mathscr{B}, \mathscr{C})$ of the wave action conservation law, when evaluated on the family of basic states (1.3), have the singularities

$$
\mathscr{B}_{k}=0, \quad \mathscr{B}_{k k}=0, \quad \text { and } \quad \mathscr{C}_{k}=0
$$

in $2+1$, then modulation with slow time and space scales

$$
X=\varepsilon x, \quad Y=\varepsilon^{2} y, \quad T=\varepsilon^{3} t,
$$

and the ansatz

$$
\widehat{U}\left(\theta+\phi, \omega+\varepsilon^{3} \Omega, k+\varepsilon q, m+\varepsilon^{2} r\right)+\varepsilon^{2} W,
$$

results in the following modulation equation for $q$

$$
\begin{aligned}
\left(\left(\mathscr{A}_{k}+\mathscr{B}_{\omega}\right) q_{T}+\frac{1}{2} \mathscr{B}_{k k k} q^{2} q_{X}\right. & \left.+\mathscr{K} q_{X X X}\right)_{X}+\mathscr{C}_{m} q_{Y Y} \\
& +\left(\mathscr{B}_{k m}+\mathscr{C}_{k k}\right)\left(q q_{Y}\right)_{X}+\mathscr{B}_{k m}\left(q_{X} \partial_{X}^{-1} q_{Y}\right)_{X}=0,
\end{aligned}
$$

with the modulation of $\Omega$ and $r$ following from conservation of waves (see (3.3) in $\S 3$ ). This equation will be called $\mathrm{mKP}-\mathrm{KD}^{+}$with the plus flagging up the second to last term. In this equation $\partial_{X}^{-1}$ denotes an antiderivative (when the antiderivative is not well-defined, the term $\partial_{X}^{-1} q_{Y}$ is replaced by $r$ ). The coefficients in (1.11) are either derivatives of the components of wave action conservation or, in the case of $\mathscr{K}$, determined by the dispersion relation or a Jordan chain argument, and the details of their construction are given in $\S 3$. 
Restriction of (1.11) to $1+1$ gives the $\mathrm{mKdV}$ equation in universal form

$$
\left(\mathcal{A}_{k}+\mathscr{B}_{\omega}\right) q_{T}+\frac{1}{2} \mathscr{B}_{k k k} q^{2} q_{X}+\mathscr{K} q_{X X X}=0,
$$

with only the first two conditions in (1.8) required. In $2+1$ one would expect that the generalisation of (1.12) is $\mathrm{mKP}$

$$
\left(\left(\mathcal{A}_{k}+\mathscr{B}_{\omega}\right) q_{T}+\frac{1}{2} \mathscr{B}_{k k k} q^{2} q_{X}+\mathscr{K} q_{X X X}\right)_{X}+\mathscr{C}_{m} q_{Y Y}
$$

Indeed, this is the appropriate generalisation when $\mathscr{B}_{k m}=0$ and this latter condition is satisfied when the basic state and equations are symmetric in $y$ (see $\S 3.1$ for precise symmetry condition). When $\mathscr{B}_{k m} \neq 0$ the latter two terms and the complete equation in (1.11) are required.

When only one of the last two terms in (1.11) is operational the mKP generalises to,

$$
\left(\left(\mathcal{A}_{k}+\mathscr{B}_{\omega}\right) q_{T}+\frac{1}{2} \mathscr{B}_{k k k} q^{2} q_{X}+\mathscr{K} q_{X X X}\right)_{X}+\mathscr{C}_{m} q_{Y Y}+\mathscr{B}_{k m}\left(q_{X} \partial_{X}^{-1} q_{Y}\right)_{X}=0 .
$$

This form of equation appears in [10,11], albeit without the representation of the coefficients in terms of the action as here, and in the literature it is called the Konopelchenko-Dubrovsky equation, or mKP-KD for short. However, the complete equation (1.11) with the $\left(q q_{Y}\right)_{X}$ term included is new. With the scaling (1.9) it is natural to include this term so it is surprising that it has not been noticed before in generalisations of mKdV to $2+1$.

The term "universal form" is used to indicate that the resulting equation is deduced from the abstract Lagrangian (1.2), and the coefficients are expressed in terms of the components of the action conservation which are defined purely in terms of the Lagrangian, and don't depend on particular PDEs. A key step in relating the properties of conservation laws to the Euler-Lagrange equation is to transform the Lagrangian into multisymplectic form.

The theory is immediately applicable to any PDE generated by a Lagrangian with a family of periodic travelling waves (or more generally a family of relative equilibria). As a consequence, the theory of this paper anticipates the emergence of the mKP-KD ${ }^{+}$equation from finite amplitude waves, rather than just trivial states, in various contexts. An example which demonstrates the application of this theory is given in $\S 4$. Starting with the cubicquintic variant of the Nonlinear Schrödinger equation (CQNLS),

$$
\mathrm{i} \Psi_{t}+\Psi_{x x}+\Psi_{y y}-\alpha|\Psi|^{2} \Psi+|\Psi|^{4} \Psi=0
$$

for the complex-valued function $\Psi(x, y, t)$ and parameter $\alpha>0$, it is shown that at particular parameter values along a branch of periodic travelling waves, the equation (1.11) emerges with $\mathscr{B}_{k m}=0$, and after scaling the coefficients, can be simplified to

$$
\left( \pm q_{T}+q^{2} q_{X}+q_{X X X}\right)_{X}-q_{Y Y}=0
$$


Dispersionless WMEs

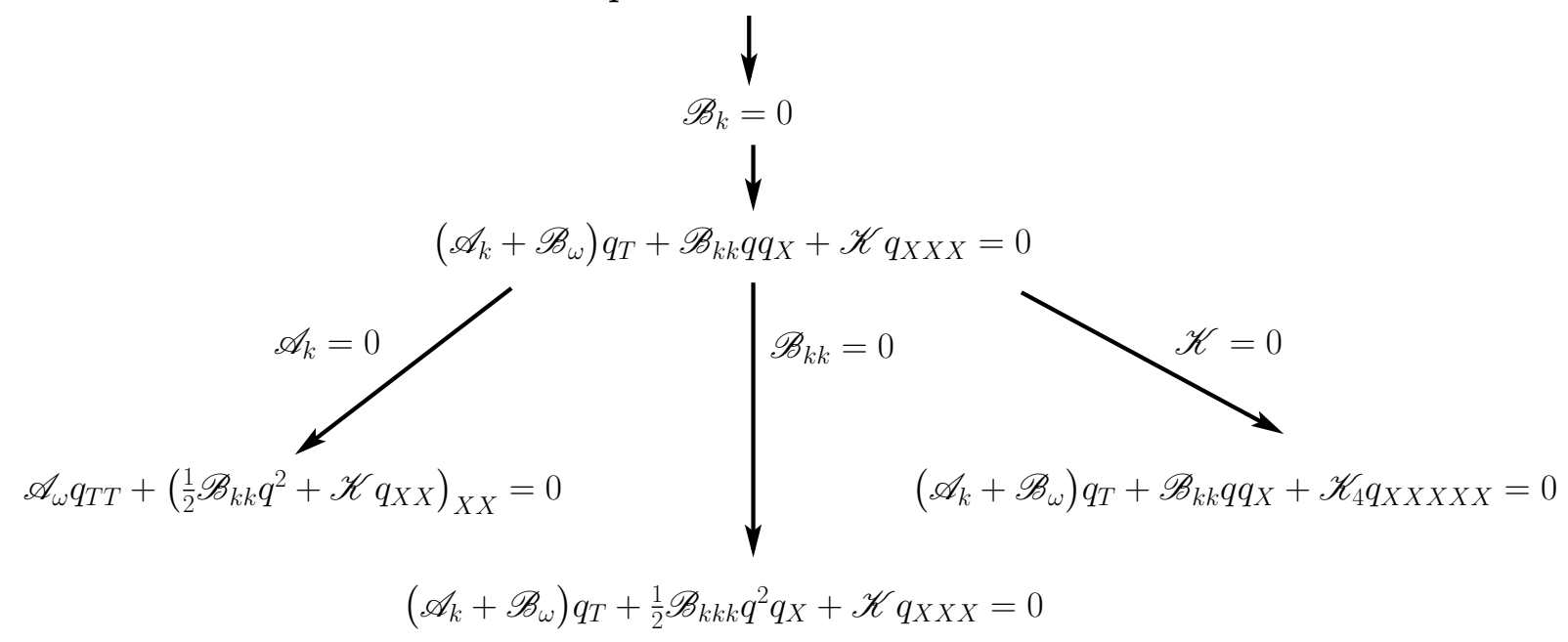

Figure 1: The three codimension two modulation equations associated with breakdown of the $\mathrm{KdV}$ equation, with the middle bottom case, $\mathscr{B}_{k k}=0$ considered in this paper.

The derivation of $\mathrm{mKdV}$ in $1+1$ in this paper completes the classification of codimension two singularities that arise from the breakdown of the $\mathrm{KdV}$ equation in phase modulation. In $1+1$, the $\mathrm{KdV}$ equation arises via phase modulation when the WMEs break down at the singularity $\mathscr{B}_{k}=0$ and in universal form [1] it is

$$
\left(\mathscr{A}_{k}+\mathscr{B}_{\omega}\right) q_{T}+\mathscr{B}_{k k} q q_{X}+\mathscr{K} q_{X X X}=0
$$

where $q(X, T)$ is the modulated wavenumber, $X=\varepsilon x$, and $T=\varepsilon^{3} t$. There are three ways this equation can degenerate producing codimension two singularities: $\mathscr{A}_{k}=0$ or $\mathscr{K}=0$ or $\mathscr{B}_{k k}=0$. In each case a new scaling and ansatz produces a different equation. A diagram showing the new codimension two equations that arise when KdV breaks down is in Figure 1. The first case, $\mathscr{A}_{k}=0$, is considered in [17] and the result is

$$
\mathscr{A}_{k}=0 \quad \text { and } \quad \mathscr{B}_{k k} \neq 0, \mathscr{K} \neq 0 \Rightarrow \mathscr{A}_{\omega} q_{T T}+\left(\frac{1}{2} \mathscr{B}_{k k} q^{2}+\mathscr{K} q_{X X}\right)_{X X}=0
$$

with the additional constraint $\mathscr{A}_{\omega} \neq 0$ generated, and new scaling. The second case, $\mathscr{K}=0$, is considered in [14] and the result is

$$
\mathscr{K}=0 \quad \text { and } \quad \mathscr{A}_{k} \neq 0, \mathscr{B}_{k k} \neq 0 \Rightarrow\left(\mathscr{A}_{k}+\mathscr{B}_{\omega}\right) q_{T}+\mathscr{B}_{k k} q q_{X}+\mathscr{K}_{5} q_{X X X X X}=0,
$$

with the additional constraint $\mathscr{K}_{5} \neq 0$ generated, and new scaling. The third case where $\mathscr{B}_{k k}=0$ but the other two coefficients in (1.17) are nonzero, and $\mathscr{B}_{k k k} \neq 0$, is considered in this paper. All three of the above have $2+1$ generalisations as well. The above "classification by codimension" is reminscent of classifications in bifurcation theory [5]. The above categorisation is in the gradient setting and so could potentially be made precise by cataloguing the singularities of the Lagrangian function $\mathscr{L}(\omega, k, m)$ using catastrophe 
theory or singularity theory for gradient bifurcation problems [2]. However in this case, since the KdV equation (1.17) has only three coefficients, the classification of codimension two singularities is immediate. However more general and higher codimensional singularities in phase dynamics will require a more systematic approach.

The format of this paper is as follows. The abstract properties of the Lagrangian relevant to the modulation theory are presented in $\S 2$, including the multisymplectic formulation, linearisation and conservation laws. The phase modulation analysis is undertaken in $\S 3$ and the equation (1.11) is derived. The theory is applied to CQNLS in $\S 4$. Implications, other directions, and generalisations are discussed in the concluding remarks $\S 5$.

\section{Lagrangian, conservation laws, and linearisation}

The starting point for the theory is PDEs generated by the $2+1$ abstract Lagrangian (1.2). The theory relies on transforming this density into multisymplectic form, and the process to do so has been documented elsewhere (e.g. [1, 17]). This transformation can be interpreted as a multi-dimensional Legendre transform, or simply as a rearrangement of the Lagrangian to isolate the derivatives in each direction. The transformed Lagrangian is

$$
\mathscr{L}(Z)=\iiint\left[\frac{1}{2}\left\langle Z, \mathbf{M} Z_{t}\right\rangle+\frac{1}{2}\left\langle Z, \mathbf{J} Z_{x}\right\rangle+\frac{1}{2}\left\langle Z, \mathbf{K} Z_{y}\right\rangle-S(Z)\right] \mathrm{d} x \mathrm{~d} y \mathrm{~d} t
$$

for a new $n$-component state vector $Z$, skew symmetric matrices $\mathbf{M}, \mathbf{J}$ and $\mathbf{K}$ and Hamiltonian function $S$. The first variation of $\mathscr{L}(Z)$, with fixed endpoints on the variations, results in an Euler-Lagrange equation of the form

$$
\mathbf{M} Z_{t}+\mathbf{J} Z_{x}+\mathbf{K} Z_{y}=\nabla S(Z)
$$

where $Z(x, y, t)$ is an $n$-component state vector. It is assumed that a periodic travelling wave solution, or relative equilibrium solution exists. For definiteness take the basic state to be the former, but the methodology proceeds almost identically for relative equilibrium. The basic state is

$$
\widehat{Z}(\theta, \omega, k, m), \quad \theta:=k x+m y+\omega t+\theta_{0},
$$

where $k, m$ are wavenumbers, $\omega$ is a frequency, and $\theta_{0}$ is a shift along the solution orbit. This solution satisfies

$$
(\omega \mathbf{M}+k \mathbf{J}+m \mathbf{K}) \widehat{Z}_{\theta}=\nabla S(\widehat{Z}) .
$$

The linearisation of (2.1) about the basic state defines the linear operator

$$
\mathbf{L} V=\mathrm{D}^{2} S(\widehat{Z}) V-(\omega \mathbf{M}+k \mathbf{J}+m \mathbf{K}) V_{\theta} .
$$

Using this definition, and differentiating (2.3), the $\theta, k, m$ and $\omega$ derivatives of the basic state satisfy

$$
\begin{array}{lr}
\mathbf{L} \widehat{Z}_{\theta}=0, & \mathbf{L} \widehat{Z}_{k}=\mathbf{J} \widehat{Z}_{\theta}, \\
\mathbf{L} \widehat{Z}_{m}=\mathbf{K} \widehat{Z}_{\theta}, & \mathbf{L} \widehat{Z}_{\omega}=\mathbf{M} \widehat{Z}_{\theta} .
\end{array}
$$


The first of these highlights that $\widehat{Z}_{\theta} \in \operatorname{ker}(\mathbf{L})$, and for the purposes of this analysis we will assume that the kernel is no larger. As a consequence solvability of inhomogeneous problems involving $\mathbf{L}$ reduces to

$$
\mathbf{L} P=Q \quad \text { is solvable when }\left\langle\left\langle\widehat{Z}_{\theta}, Q\right\rangle\right\rangle=0,
$$

for an inner product $\langle\langle\cdot, \cdot\rangle\rangle$. For the periodic travelling wave basic state the inner product is

$$
\langle\langle P, Q\rangle\rangle:=\frac{1}{2 \pi} \int_{0}^{2 \pi}\langle P, Q\rangle \mathrm{d} \theta
$$

where $\langle\cdot, \cdot\rangle$ is the Euclidean inner product on $\mathbb{R}^{n}$.

\subsection{Conservation laws}

The theory of phase modulation relies on multisymplectic Noether theory, which relates symmetry to conservation laws using the matrices $\mathbf{M}, \mathbf{J}$ and $\mathbf{K}$. In the present context, where the basic state is a periodic travelling wave, the conservation law of interest is conservation of wave action $[1,16,17]$. After evaluating the components of wave action on the basic state and averaging, they are

$$
\mathscr{A}(\omega, k, m)=\frac{1}{2}\left\langle\left\langle\widehat{Z}, \mathbf{M} \widehat{Z}_{\theta}\right\rangle\right\rangle, \quad \mathscr{B}(\omega, k, m)=\frac{1}{2}\left\langle\left\langle\widehat{Z}, \mathbf{J} \widehat{Z}_{\theta}\right\rangle\right\rangle, \quad \mathscr{C}(\omega, k, m)=\frac{1}{2}\left\langle\left\langle\widehat{Z}, \mathbf{K} \widehat{Z}_{\theta}\right\rangle\right\rangle .
$$

Derivatives of these arise in the theory, and form both necessary emergence conditions and some of the coefficients in the emergent PDE. The derivatives which are needed in the theory are:

$$
\begin{aligned}
\mathscr{A}_{k} & =\left\langle\left\langle\widehat{Z}_{k}, \mathbf{M} \widehat{Z}_{\theta}\right\rangle\right\rangle=\mathscr{B}_{\omega}, \\
\mathscr{B}_{k} & =\left\langle\left\langle\widehat{Z}_{k}, \mathbf{J} \widehat{Z}_{\theta}\right\rangle\right\rangle, \\
\mathscr{C}_{k} & =\left\langle\left\langle\widehat{Z}_{k}, \mathbf{K} \widehat{Z}_{\theta}\right\rangle\right\rangle=\mathscr{B}_{m}, \\
\mathscr{B}_{k k} & =\left\langle\left\langle\widehat{Z}_{k k}, \mathbf{J} \widehat{Z}_{\theta}\right\rangle\right\rangle+\left\langle\left\langle\widehat{Z}_{k}, \mathbf{J} \widehat{Z}_{\theta k}\right\rangle\right\rangle, \\
\mathscr{C}_{k k} & =\left\langle\left\langle\widehat{Z}_{k k}, \mathbf{K} \widehat{Z}_{\theta}\right\rangle\right\rangle+\left\langle\left\langle\widehat{Z}_{k}, \mathbf{C} \widehat{Z}_{\theta k}\right\rangle\right\rangle=\mathscr{B}_{k m}, \\
\mathscr{B}_{k k k} & =\left\langle\left\langle\widehat{Z}_{k k k}, \mathbf{J} \widehat{Z}_{\theta}\right\rangle\right\rangle+3\left\langle\left\langle\widehat{Z}_{k k}, \mathbf{J} \widehat{Z}_{\theta k}\right\rangle\right\rangle .
\end{aligned}
$$

\subsection{Jordan Chain Theory}

Aside from the conservation laws, the other main structure which arises within the reduction scheme is that of a Jordan chain. Ultimately, this leads to a characterisation of the dispersion term in the reduced PDE (1.11). The relevant theory needed is as follows. We will be brief as the necessary theory for Jordan chains has been given in our previous papers $[1,16,17]$.

The first two equations in (2.4) highlight the presence of a Jordan chain of the form

$$
\mathbf{L} \xi_{1}=0, \quad \mathbf{L} \xi_{i+1}=\mathbf{J} \xi_{i}, i>0,
$$


with $\xi_{1}=\widehat{Z}_{\theta}, \xi_{2}=\widehat{Z}_{k}$. Using the notion of solvability introduced earlier, a third element will exist when

$$
0=\left\langle\left\langle\widehat{Z}_{\theta}, \mathbf{J} \widehat{Z}_{k}\right\rangle\right\rangle=-\mathscr{B}_{k},
$$

by using (2.6). When this derivative vanishes the chain increases in length, allowing for the definition

$$
\mathbf{L} \xi_{3}=\mathbf{J} \xi_{2}=\mathbf{J} \widehat{Z}_{k}
$$

A fourth element is automatic since $\mathbf{L} \xi_{2}=\mathbf{J} \xi_{3}$ is solvable:

$$
\left\langle\left\langle\widehat{Z}_{\theta}, \mathbf{J} \xi_{3}\right\rangle\right\rangle=-\left\langle\left\langle\widehat{Z}_{k}, \mathbf{J} \widehat{Z}_{k}\right\rangle\right\rangle=0 .
$$

This paper assumes that the chain terminates at length four, that is; $\mathbf{L} \xi_{5}=\mathbf{J} \xi_{4}$ is not solvable, and so we introduce the quantity

$$
\mathscr{K}=\left\langle\left\langle\mathbf{J} \widehat{Z}_{\theta}, \xi_{4}\right\rangle\right\rangle \neq 0 .
$$

It can be simplified using the Jordan chain

$$
\mathscr{K}:=\left\langle\left\langle\mathbf{J} \widehat{Z}_{\theta}, \xi_{4}\right\rangle\right\rangle=\left\langle\left\langle\mathbf{L} \xi_{2}, \xi_{4}\right\rangle\right\rangle=\left\langle\left\langle\xi_{2}, \mathbf{L} \xi_{4}\right\rangle\right\rangle=\left\langle\left\langle\xi_{2}, \mathbf{J} \xi_{3}\right\rangle\right\rangle .
$$

This quantity will show up as a dispersion coefficient in the phase modulation theory.

\section{Reduction to $\mathrm{mKP}-\mathrm{KD}^{+}$via phase modulation}

The following modulation ansatz is proposed

$$
Z(x, y, t)=\widehat{Z}\left(\theta+\phi, k+\varepsilon q, m+\varepsilon^{2} r, \omega+\varepsilon^{3} \Omega\right)+\varepsilon^{2} W(\theta+\phi, X, Y, T),
$$

where $\phi, q, r, \Omega$ are functions of the slow variables $(X, Y, T)$, and $\varepsilon$, with definitions

$$
X=\varepsilon x, \quad Y=\varepsilon^{2} y, \quad T=\varepsilon^{3} t .
$$

That is is the correct ansatz is confirmed a posteriori. The modulation frequency and wavenumbers are realated by the conservation of waves,

$$
\Omega=\phi_{T}, \quad q=\phi_{X}, \quad r=\phi_{Y},
$$

which by cross-differentation give

$$
q_{T}=\Omega_{X}, \quad r_{T}=\Omega_{Y}, \quad r_{X}=q_{Y} .
$$

The idea is to the substitute the ansatz (3.1) into (2.1), Taylor expand around $\varepsilon=0$ and solve the resulting system of equations. The remainder term $W$ is also expanded in an asymptotic series, with

$$
W=W_{0}(\theta+\phi, X, Y, T)+\varepsilon W_{1}(\theta+\phi, X, Y, T)+\varepsilon^{2} W_{2}(\theta+\phi, X, Y, T)+\ldots .
$$


A key difference from previous analyses of this type is that the remainder term $W$ now depends on $\phi$, since the perturbation $\theta \mapsto \theta+\phi$ is of order unity.

The equations at order $\varepsilon^{j}, j=0,1,2,3,4$ are needed, with the first three brief, and the $\mathrm{mkP}-\mathrm{KD}^{+}$arising at fourth order. The $\varepsilon^{0}$ equations is just

$$
(\omega \mathbf{M}+k \mathbf{J}+m \mathbf{K}) \widehat{Z}_{\theta}=\nabla S(\widehat{Z}),
$$

recovering (2.3) and $\widehat{Z}$ satisfies this equation by assumption. Using the second result of (2.4), the $\varepsilon^{1}$ equation reduces to

$$
\left(q-\phi_{X}\right) \mathbf{L} \widehat{Z}_{\theta}=0
$$

recovering the phase consistency condition $q=\phi_{X}$. Ignoring terms that cancel and using (2.4) again, the $\varepsilon^{2}$ equation is

$$
\mathbf{L} W_{0}+\left(r-\phi_{Y}\right) \mathbf{L} \widehat{Z}_{m}=q_{X} \mathbf{J} \widehat{Z}_{k}
$$

The second term on the left vanishes trivially by the phase consistency condition $r=\phi_{Y}$. For the term on the right hand side, solvability is imposed and generates the condition

$$
0=\left\langle\left\langle\widehat{Z}_{\theta}, \mathbf{J} \widehat{Z}_{k}\right\rangle\right\rangle=-\left\langle\left\langle\mathbf{J} \widehat{Z}_{\theta}, \widehat{Z}_{k}\right\rangle\right\rangle=-\mathscr{B}_{k}
$$

This is precisely the condition seen in $\S 2.2$ for the chain to be of length 4 . Assuming the condition $\mathscr{B}_{k}=0$, the equation can be solved for $W_{0}$, giving

$$
W_{0}=q_{X} \xi_{3}
$$

where $\xi_{3}$ has been defined in (2.7), plus an arbitrary amount of homogeneous solution, which drops out in the final analysis.

At third order the equations get a bit more complicated. The $\varepsilon^{3}$ terms are

$$
\begin{array}{r}
\mathbf{L} W_{1}+\left(\Omega-\phi_{T}\right) \mathbf{L} \widehat{Z}_{\omega}=\quad q q_{X}\left(\mathbf{J} \widehat{Z}_{k k}+\mathbf{J} \partial_{\theta} \xi_{3}-\mathrm{D}^{3} S(\widehat{Z})\left(\widehat{Z}_{k}, \xi_{3}\right)\right) \\
+q_{Y}\left(\mathbf{K} \widehat{Z}_{k}+\mathbf{J} \widehat{Z}_{m}\right)+q_{X X} \mathbf{J} \xi_{3}
\end{array}
$$

Since $\Omega=\phi_{T}$ the second term on the left hand side vanishes. Now, consider the solvability of the right hand side. The $q_{Y}$ term is solvable when

$$
0=\left\langle\left\langle\widehat{Z}_{\theta}, \mathbf{K} \widehat{Z}_{k}+\mathbf{J} \widehat{Z}_{m}\right\rangle\right\rangle=-\mathscr{B}_{m}-\mathscr{C}_{k} .
$$

We assume this condition to be satisfied henceforth in this analysis. The term in $q_{X X}$ is automatically solvable, using the fourth element in the Jordan chain, $\mathbf{L} \xi_{4}=\mathbf{J} \xi_{3}$. The final term we consider is the $q q_{X}$ term. Assessing solvability,

$$
\begin{aligned}
0 & =\left\langle\left\langle\widehat{Z}_{\theta}, \mathbf{J} \widehat{Z}_{k k}\right\rangle\right\rangle+\left\langle\left\langle\widehat{Z}_{\theta}, \mathbf{J} \partial_{\theta} \xi_{3}-\mathrm{D}^{3} S(\widehat{Z})\left(\widehat{Z}_{k}, \xi_{3}\right)\right\rangle\right\rangle \\
& =\left\langle\left\langle\widehat{Z}_{\theta}, \mathbf{J} \widehat{Z}_{k k}\right\rangle\right\rangle+\left\langle\left\langle\xi_{3}, \mathbf{L} \widehat{Z}_{\theta k}\right\rangle\right\rangle \\
& =\left\langle\left\langle\widehat{Z}_{\theta}, \mathbf{J} \widehat{Z}_{k k}\right\rangle\right\rangle+\left\langle\left\langle\widehat{Z}_{\theta k}, \mathbf{J} \widehat{Z}_{k}\right\rangle\right\rangle=-\mathscr{B}_{k k} .
\end{aligned}
$$


Therefore we require $\mathscr{B}_{k k}$ to vanish for the analysis to continue. This confirms the necessity of all three conditions (1.8) for the emergence of mKP-KD ${ }^{+}$.

When the conditions are met, the solution for $W_{1}$ is

$$
W_{1}=q q_{X} \partial_{k} \xi_{3}+q_{X X} \xi_{4}+r_{X} \zeta
$$

plus an arbitrary amount of homogeneous solution which is neglected as it does not affect the final equation at fourth order. In (3.7) the function $\zeta$ is defined by

$$
\mathbf{L} \zeta=\mathbf{K} \widehat{Z}_{k}+\mathbf{J} \widehat{Z}_{m}
$$

The function $\partial_{k} \xi_{3}$ in the $q q_{X}$ term has been deduced by taking the $k$ derivative of the defining equation for $\xi_{3}$,

$$
\mathrm{D}^{3} S(\widehat{Z})\left(\widehat{Z}_{k}, \xi_{3}\right)+\mathrm{D}^{2} S(\widehat{Z}) \partial_{k} \xi_{3}-(\omega \mathbf{M}+k \mathbf{J}) \partial_{\theta k} \xi_{3}-\mathbf{J}\left(\xi_{3}\right)_{\theta}=\mathbf{J} \widehat{Z}_{k k},
$$

or

$$
\mathbf{L} \partial_{k} \xi_{3}=\mathbf{J} \widehat{Z}_{k k}+\mathbf{J} \partial_{\theta} \xi_{3}-\mathrm{D}^{3} S(\widehat{Z})\left(\widehat{Z}_{k}, \xi_{3}\right) .
$$

At fourth order, collecting the necessary terms and simplifying the result,

$$
\begin{aligned}
& \mathbf{L} W_{2}=q_{T}\left.\mathbf{M} \widehat{Z}_{k}+\mathbf{J} \widehat{Z}_{\omega}\right)+q_{X X X} \mathbf{J} \xi_{4}+q_{X Y}\left(\mathbf{J} \zeta_{1}+\mathbf{K} \xi_{3}\right)+r_{Y} \mathbf{K} \widehat{Z}_{m} \\
&+ q r_{X}\left(\mathbf{J} \widehat{Z}_{k m}+\mathbf{K} \widehat{Z}_{k k}+\mathbf{J} \zeta_{1}-\mathrm{D}^{3} S(\widehat{Z})\left(\zeta_{1}, \widehat{Z}_{k}\right)\right) \\
&+ r q_{X}\left(\mathbf{J} \widehat{Z}_{k m}+\mathbf{K} \partial_{\theta} \xi_{3}-\mathrm{D}^{3} S(\widehat{Z})\left(\widehat{Z}_{m}, \xi_{3}\right)\right) \\
&+ q q_{X X}\left(\mathrm{D}^{3} S(\widehat{Z})\left(\widehat{Z}_{k}, \xi_{4}\right)-\mathbf{J}\left(\xi_{4}\right)_{\theta}-\mathbf{J} \partial_{k} \xi_{3}\right)+\left(q_{X}\right)^{2}\left(\mathrm{D}^{3} S(\widehat{Z})\left(\xi_{3}, \xi_{3}\right)-2 \mathbf{J} \partial_{k} \xi_{3}\right) \\
&+q^{2} q_{X}\left(\frac{1}{2} \mathbf{J} \widehat{Z}_{k k k}+\mathbf{J}(\xi)_{\theta k}-\frac{1}{2} \mathrm{D}^{4} S(\widehat{Z})\left(\widehat{Z}_{k}, \widehat{Z}_{k}, \xi_{3}\right)\right) \\
& \quad-q^{2} q_{X}\left(\frac{1}{2} \mathrm{D}^{3} S(\widehat{Z})\left(\widehat{Z}_{k k}, \xi_{3}\right)+\mathrm{D}^{3} S(\widehat{Z})\left(\widehat{Z}_{k}, \partial_{k} \xi_{3}\right)\right) .
\end{aligned}
$$

Solvability of the above is now considered, with the final result being the desired phase equation. Take the inner product of the right hand side with $\widehat{Z}_{\theta}=\xi_{1}$. Firstly, the coefficient of the $q_{T}$ term can be computed to show that

$$
\left\langle\left\langle\widehat{Z}_{\theta}, \mathbf{M} \widehat{Z}_{k}+\mathbf{J} \widehat{Z}_{\omega}\right\rangle\right\rangle=-\mathscr{A}_{k}-\mathscr{B}_{k} .
$$

Using the definition (2.8), the coefficient of the $q_{X X X}$ term is

$$
\left\langle\left\langle\widehat{Z}_{\theta}, \mathbf{J} \xi_{4}\right\rangle\right\rangle=-\mathscr{K} .
$$

Similarly the coefficient of $r_{Y}$ is

$$
\left\langle\left\langle\widehat{Z}_{\theta}, \mathbf{K} \widehat{Z}_{m}\right\rangle\right\rangle=-\mathscr{C}_{m}
$$


The next considered is the $q^{2} q_{X}$ term. Starting from our expression for $\mathscr{B}_{k k}$,

$$
\begin{aligned}
\mathscr{B}_{k k k}= & \partial_{k}\left(\mathscr{B}_{k k}\right) \\
= & \partial_{k}\left(-\left\langle\left\langle\widehat{Z}_{\theta}, \mathbf{J} \widehat{Z}_{k k}\right\rangle\right\rangle-\left\langle\left\langle\widehat{Z}_{\theta}, \mathbf{J} \partial_{\theta} \xi_{3}\right\rangle\right\rangle+\left\langle\left\langle\widehat{Z}_{\theta}, D^{3} S(\widehat{Z})\left(\widehat{Z}_{k}, \xi_{3}\right)\right\rangle\right)\right. \\
= & -\left\langle\left\langle\widehat{Z}_{\theta}, \mathbf{J} \widehat{Z}_{k k k}+\mathbf{J} \partial_{\theta k} \xi_{3}-D^{4} S(\widehat{Z})\left(\widehat{Z}_{k}, \widehat{Z}_{k}, \xi_{3}\right)-D^{3} S(\widehat{Z})\left(\widehat{Z}_{k k}, \xi_{3}\right)\right\rangle\right\rangle \\
& \quad+\left\langle\left\langle\widehat{Z}_{\theta}, D^{3} S(\widehat{Z})\left(\widehat{Z}_{k}, \partial_{k} \xi_{3}\right)\right\rangle\right\rangle-\left\langle\left\langle\widehat{Z}_{\theta k}, \mathbf{L} \partial_{k} \xi_{3}\right\rangle\right\rangle .
\end{aligned}
$$

This last term can be manipulated to obtain

$$
\left\langle\left\langle\widehat{Z}_{\theta k}, \mathbf{L} \partial_{k} \xi_{3}\right\rangle\right\rangle=\left\langle\left\langle\mathbf{J} \widehat{Z}_{\theta \theta}-\mathrm{D}^{3} S(\widehat{Z})\left(\widehat{Z}_{k}, \widehat{Z}_{\theta}\right), \partial_{k} \xi_{3}\right\rangle\right\rangle=\left\langle\left\langle\widehat{Z}_{\theta}, \mathbf{J} \partial_{\theta k} \xi_{3}\right\rangle\right\rangle-\left\langle\left\langle\widehat{Z}_{\theta}, \mathrm{D}^{3} S(\widehat{Z})\left(\widehat{Z}_{k}, \partial_{k} \xi_{3}\right)\right\rangle\right\rangle,
$$

and so

$\mathscr{B}_{k k k}=\left\langle\left\langle\widehat{Z}_{\theta}, \mathrm{D}^{4} S(\widehat{Z})\left(\widehat{Z}_{k}, \widehat{Z}_{k}, \xi_{3}\right)+\mathrm{D}^{3} S(\widehat{Z})\left(\widehat{Z}_{k k}, \xi_{3}\right)+2 \mathrm{D}^{3} S(\widehat{Z})\left(\widehat{Z}_{k}, \partial_{k} \xi_{3}\right)-\mathbf{J} \widehat{Z}_{k k k}-2 \mathbf{J} \partial_{\theta k} \xi_{3}\right\rangle\right\rangle$.

This shows that the coefficient of the $q^{2} q_{X}$ term is $-\frac{1}{2} \mathscr{B}_{k k k}$.

Now consider the quadratic terms. Starting with the $q q_{X X}$ term, its coefficient is given by

$$
\begin{aligned}
& \left\langle\left\langle\widehat{Z}_{\theta}, \mathrm{D}^{3} S(\widehat{Z})\left(\widehat{Z}_{k}, \xi_{4}\right)-\mathbf{J} \partial_{\theta} \xi_{4}-\mathbf{J} \partial_{k} \xi_{3}\right\rangle\right\rangle \\
= & \left\langle\left\langle\mathbf{J} \widehat{Z}_{\theta}, \partial_{k} \xi_{3}\right\rangle\right\rangle+\left\langle\left\langle\mathbf{J} \widehat{Z}_{\theta}, \partial_{\theta} \xi_{4}\right\rangle\right\rangle+\left\langle\left\langle\widehat{Z}_{k}, \mathrm{D}^{3} S(\widehat{Z})\left(\widehat{Z}_{\theta}, \xi_{4}\right)\right\rangle\right\rangle \\
= & \left\langle\left\langle\mathbf{J} \widehat{Z}_{\theta}, \partial_{k} \xi_{3}\right\rangle\right\rangle+\left\langle\left\langle\widehat{Z}_{k}, \mathbf{J} \partial_{\theta} \xi_{3}-\mathrm{D}^{3} S(\widehat{Z})\left(\widehat{Z}_{\theta}, \xi_{4}\right)\right\rangle\right\rangle+\left\langle\left\langle\widehat{Z}_{k}, D^{3} S(\widehat{Z})\left(\widehat{Z}_{\theta}, \xi_{4}\right)\right\rangle\right\rangle \\
= & \left\langle\left\langle\mathbf{J} \widehat{Z}_{\theta}, \partial_{k} \xi_{3}\right\rangle\right\rangle+\left\langle\left\langle\widehat{Z}_{k}, \mathbf{J} \partial_{\theta} \xi_{3}\right\rangle\right\rangle \\
= & \partial_{k}\left[\left\langle\left\langle\mathbf{J} \widehat{Z}_{\theta}, \xi_{3}\right\rangle\right\rangle\right] .
\end{aligned}
$$

In the above, we have used that

$$
\mathbf{L} \partial_{\theta} \xi_{4}=\mathbf{J} \partial_{\theta} \xi_{3}-\mathrm{D}^{3} S(\widehat{Z})\left(\widehat{Z}_{\theta}, \xi_{4}\right),
$$

which can be seen by differentiating the defining equation for $\xi_{4}$. The expression inside the bracket is zero since

$$
\left\langle\left\langle\mathbf{J} \widehat{Z}_{\theta}, \xi_{3}\right\rangle\right\rangle=-\left\langle\left\langle\widehat{Z}_{\theta}, \mathbf{J} \xi_{3}\right\rangle\right\rangle=-\left\langle\left\langle\widehat{Z}_{\theta}, \mathbf{L} \xi_{4}\right\rangle\right\rangle=-\left\langle\left\langle\mathbf{L} \widehat{Z}_{\theta}, \xi_{4}\right\rangle\right\rangle=0 .
$$

The $q_{X}^{2}$ term is shown to vanish in a similar way,

$$
\begin{aligned}
& \left\langle\left\langle\widehat{Z}_{\theta}, \mathrm{D}^{3} S(\widehat{Z})\left(\xi_{3}, \xi_{3}\right)-2 \mathbf{J} \partial_{k} \xi_{3}\right\rangle\right\rangle \\
= & \left\langle\left\langle\xi_{3}, \mathrm{D}^{3} S(\widehat{Z})\left(\widehat{Z}_{\theta}, \xi_{3}\right)\right\rangle\right\rangle-2\left\langle\left\langle\widehat{Z}_{k}, \mathbf{J} \partial_{\theta} \xi_{3}\right\rangle\right\rangle \\
= & \left\langle\left\langle\xi_{3}, \mathrm{D}^{3} S(\widehat{Z})\left(\widehat{Z}_{\theta}, \xi_{3}\right)-2 \mathbf{J} \widehat{Z}_{k \theta}\right\rangle\right\rangle \\
= & -\left\langle\left\langle\xi_{3}, \mathbf{L} \partial_{\theta} \xi_{3}+\mathbf{J} \widehat{Z}_{\theta k}\right\rangle\right\rangle \\
= & -\left\langle\left\langle\mathbf{J} \widehat{Z}_{k}, \partial_{\theta} \xi_{3}\right\rangle\right\rangle-\left\langle\left\langle\xi_{3}, \mathbf{J} \widehat{Z}_{\theta k}\right\rangle\right\rangle \\
= & -\left\langle\left\langle\mathbf{J} \widehat{Z}_{k}, \partial_{\theta} \xi_{3}\right\rangle\right\rangle+\left\langle\left\langle\partial_{\theta} \xi_{3}, \mathbf{J} \widehat{Z}_{k}\right\rangle\right\rangle \\
= & 0
\end{aligned}
$$


using results from (3.11) and (3.13) in going from the second to third lines. Therefore both terms, $q q_{X X}$ and $q_{X}^{2}$, which have the correct scaling but are not conservative, disappear in the solvability condition.

Next consider the mixed terms (with both $r$ and $q$ ). Firstly, the $q r_{X}$ term simplifies to

$$
\begin{aligned}
& \left\langle\left\langle\widehat{Z}_{\theta}, \mathbf{J} \widehat{Z}_{k m}+\mathbf{K} \widehat{Z}_{k k}+\mathbf{J} \partial_{\theta} \eta-\mathrm{D}^{3} S(\widehat{Z})\left(\eta, \widehat{Z}_{k}\right)\right\rangle\right\rangle \\
= & \left\langle\left\langle\widehat{Z}_{\theta}, \mathbf{J} \widehat{Z}_{k m}+\mathbf{K} \widehat{Z}_{k k}\right\rangle\right\rangle+\left\langle\left\langle\eta, \mathbf{J} \widehat{Z}_{\theta \theta}-\mathrm{D}^{3} S(\widehat{Z})\left(\widehat{Z}_{\theta}, \widehat{Z}_{k}\right)\right\rangle\right\rangle \\
= & \left\langle\left\langle\widehat{Z}_{\theta}, \mathbf{J} \widehat{Z}_{k m}+\mathbf{K} \widehat{Z}_{k k}\right\rangle\right\rangle+\left\langle\left\langle\mathbf{J} \widehat{Z}_{m}+\mathbf{K} \widehat{Z}_{k}, \widehat{Z}_{\theta k}\right\rangle\right\rangle \\
= & -\left(\left\langle\left\langle\widehat{Z}, \mathbf{J} \widehat{Z}_{\theta k m}\right\rangle\right\rangle+\left\langle\left\langle\widehat{Z}_{m}, \mathbf{J} \widehat{Z}_{\theta k}\right\rangle\right\rangle\right)-\left(\left\langle\left\langle\widehat{Z}, \mathbf{K} \widehat{Z}_{\theta k k}\right\rangle\right\rangle+\left\langle\left\langle\widehat{Z}_{k}, \mathbf{K} \widehat{Z}_{\theta k}\right\rangle\right\rangle\right) \\
= & \left.-\mathscr{B}_{k m}-\mathscr{C}_{k k} \quad \text { (noting that } \mathscr{B}_{k m}=\mathscr{C}_{k k}\right) .
\end{aligned}
$$

Similarly, for the $r q_{X}$ term,

$$
\begin{aligned}
& \left\langle\left\langle\widehat{Z}_{\theta}, \mathbf{J} \widehat{Z}_{k m}+\mathbf{K} \partial_{\theta} \xi_{3}-\mathrm{D}^{3} S(\widehat{Z})\left(\xi_{3}, \widehat{Z}_{m}\right)\right\rangle\right\rangle \\
= & \left\langle\left\langle\widehat{Z}_{\theta}, \mathbf{J} \widehat{Z}_{k m}\right\rangle\right\rangle+\left\langle\left\langle\xi_{3}, \mathbf{K} \widehat{Z}_{\theta \theta}-\mathrm{D}^{3} S(\widehat{Z})\left(\widehat{Z}_{\theta}, \widehat{Z}_{m}\right)\right\rangle\right\rangle \\
= & \left\langle\left\langle\widehat{Z}_{\theta}, \mathbf{J} \widehat{Z}_{k m}\right\rangle\right\rangle+\left\langle\left\langle\mathbf{J} \widehat{Z}_{k}, \widehat{Z}_{\theta m}\right\rangle\right\rangle \\
= & \left.-\left(\left\langle\widehat{Z}, \mathbf{J} \widehat{Z}_{\theta k m}\right\rangle\right\rangle+\left\langle\left\langle\widehat{Z}_{m}, \mathbf{J} \widehat{Z}_{\theta k}\right\rangle\right)\right) \\
= & -\mathscr{B}_{k m} .
\end{aligned}
$$

Combining the results of the above calculations, we have shown that the equation for $W_{2}$ in (3.9) is solvable when $q, r$ satisfy the PDE

$$
\left(\mathscr{A}_{k}+\mathscr{B}_{\omega}\right) q_{T}+\frac{1}{2} \mathscr{B}_{k k k} q^{2} q_{X}+\mathscr{K} q_{X X X}+\mathscr{C}_{m} r_{Y}+\left(\mathscr{B}_{k m}+\mathscr{C}_{k k}\right) q r_{X}+\mathscr{B}_{k m} r q_{X}=0 .
$$

Differentiating with respect to $X$ and introducing the antiderivative to solve $r_{X}=q_{Y}$ for $r$ recovers (1.11). Alternately, expressing $q=\phi_{X}$ and $r=\phi_{Y}$ in terms of the phase in 3.14), the pure phase version of (1.11) is

$$
\left(\left(\mathscr{A}_{k}+\mathscr{B}_{\omega}\right) \phi_{T}+\frac{1}{6} \mathscr{B}_{k k k} \phi_{X}^{3}+\mathscr{K} \phi_{X X X}\right)_{X}+\mathscr{C}_{m} \phi_{Y Y}+\left(\mathscr{B}_{k m}+\mathscr{C}_{k k}\right) \phi_{X} \phi_{X Y}+\mathscr{B}_{k m} \phi_{Y} \phi_{X X}=0 .
$$

\subsection{Reflection symmetric basic state}

If the equation has a reflection symmetry in the $y$-direction and the basic state inherits this symmetry, it is shown in $\S 7$ of [16] that the third component of the wave action conservation law in (2.5) satisfies

$$
\mathscr{C}(k,-m, \omega)=-\mathscr{C}(\omega, k, m) .
$$

With this symmetry it is immediate that

$$
\left.\mathscr{C}_{k}\right|_{m=0}=\left.\mathscr{C}_{k k}\right|_{m=0}=0 .
$$


Hence when the basic state is aligned with the $x$-axis $(m=0)$ the third necessary condition in (1.8) is automatically satisfied, and the coefficents in the latter two terms in (1.11) vanish simplifying the emergent phase equations to the mKP equation

$$
\left(\left(\mathscr{A}_{k}+\mathscr{B}_{\omega}\right) q_{T}+\frac{1}{2} \mathscr{B}_{k k k} q^{2} q_{X}+\mathscr{K} q_{X X X}\right)_{X}+\mathscr{C}_{m} q_{Y Y}=0
$$

with the only necessary conditions being $\mathscr{B}_{k}=\mathscr{B}_{k k}=0$.

\section{Example: reduction of CQNLS to mKP}

The cubic-quintic NLS (CQNLS) equation in standard form is given in (1.15). It appears in the optics literature, describing the propagation of light pulses [8], and in the literature on Bose-Einstein condensates [9]. A self-consistent derivation of CQNLS is given [13]. The CQNLS equation is the Euler-Lagrange equation associated with the Lagrangian

$$
\mathcal{L}=\int_{t_{1}}^{t_{2}} \int_{x_{1}}^{x_{2}}\left[\frac{1}{2} \mathrm{i}\left(\bar{\Psi} \Psi_{t}-\Psi \bar{\Psi}_{t}\right)-\left|\Psi_{x}\right|^{2}-\left|\Psi_{y}\right|^{2}-\frac{1}{2} \alpha|\Psi|^{4}+\frac{1}{3}|\Psi|^{6}\right] \mathrm{d} x \mathrm{~d} t
$$

for real constant $\alpha$, leading to the equation

$$
i \Psi_{t}+\nabla^{2} \Psi-\alpha|\Psi|^{2} \Psi+|\Psi|^{4} \Psi=0 .
$$

The CQNLS equation (4.2) has a three-parameter family of exact periodic travelling wave solutions

$$
\Psi(x, y, t)=\Psi_{0} \mathrm{e}^{\mathrm{i} \theta}, \quad \theta=k x+m y+\omega t .
$$

Substitution of this form of solution into (4.2) gives the amplitude as a function of the wavenumber and frequency,

$$
\Delta:=\left|\Psi_{0}\right|^{4}-\alpha\left|\Psi_{0}\right|^{2}-\omega-k^{2}-m^{2}=0 .
$$

Rearranging, $\Delta=0$ is equivalent to

$$
\left(\left|\Psi_{0}\right|^{2}-\frac{1}{2} \alpha\right)^{2}-k^{2}=\omega+m^{2}+\frac{1}{4} \alpha^{2},
$$

giving the necessary conditions for existence

$$
\left|\Psi_{0}\right|^{2}>0 \text { and } \omega+k^{2}+m^{2}+\frac{1}{4} \alpha^{2}>0 .
$$

In the $\left(k,\left|\Psi_{0}\right|\right)$ plane the set $\Delta^{-1}(0)$ is a family of hyperbola parameterised for each fixed $(m, \alpha)$ by $\omega$.

The class of solutions (4.3) can also be considered as a relative equilibrium associated with the $S O(2)$ symmetry; that is, $\mathrm{e}^{\mathrm{i} s} \Psi(x, y, t)$ is a solution of $(4.2)$ whenever $\Psi(x, y, t)$ is a solution, for any $s \in \mathbb{R}$. The conservation law for this symmetry is $A_{t}+B_{x}+C_{y}=0$ with

$$
A=\frac{1}{2}|\Psi|^{2}, \quad B=\frac{1}{2}\left(\Psi^{*} \Psi_{x}-\Psi_{x}^{*} \Psi\right), \quad C=\frac{1}{2}\left(\Psi^{*} \Psi_{y}-\Psi_{y}^{*} \Psi\right),
$$


where $*$ denotes complex conjugation. This conservation law can be confirmed using Noether theory or by direct calculation. Evaluating the components of the conservation law on the family of basic states gives

$$
\mathscr{A}=\frac{1}{2}\left|\Psi_{0}\right|^{2}, \quad \mathscr{B}=k\left|\Psi_{0}\right|^{2}, \quad \mathscr{C}=m\left|\Psi_{0}\right|^{2},
$$

with $\left|\Psi_{0}\right|^{2}$ expressing using (4.4) as a function of $(\omega, k, m)$. In looking for singular points, in the family of periodic travelling waves, where mKdV emerges, one observation is immediate: $\mathscr{C}(\omega, k, m)$ is an odd function of $m$, since $\left|\Psi_{0}\right|^{2}$ is an even function of $m$, and so

$$
\left.\mathscr{C}_{k}\right|_{m=0}=\left.\mathscr{C}_{k k}\right|_{m=0}=0 .
$$

Therefore, set $m=0$, and then we need only identify values of $(\omega, k)$ where $\mathscr{B}_{k}=0$ and $\mathscr{B}_{k k}=0$, and the emergent equation will be mKP rather than mKP-KD or mKP-KD ${ }^{+}$due to the fact that $\mathscr{C}_{k k}=\mathscr{B}_{k m}=0$.

Differentiating $\mathscr{B}$ twice gives

$$
\mathscr{B}_{k}=u+k u_{k} \quad \text { and } \quad \mathscr{B}_{k k}=2 u_{k}+u_{k k}, \quad \text { with } u:=\left|\Psi_{0}\right|^{2},
$$

and

$$
(2 u-\alpha) u_{k}=2 k \text { and }(2 u-\alpha) u_{k k}+2 u_{k}^{2}=2 .
$$

Combining the existence condition (4.4), the condition $\mathscr{B}_{k}=0$ and the condition $\mathscr{B}_{k k}=0$, and setting $m=0$, leads to the three simultaneous nonlinear equations. These equations can be best visualised as three curves in the $(u, k, \omega)$ plane,

$$
\begin{aligned}
\Delta=0 & & \left(u-\frac{1}{2} \alpha\right)^{2}-k^{2}=\omega+\frac{1}{4} \alpha^{2} \\
\mathscr{B}_{k}=0 & & \left(u-\frac{1}{4} \alpha\right)^{2}+k^{2}=\frac{1}{16} \alpha^{2} \\
\mathscr{B}_{k k}=0 & & \left(u-\frac{1}{2} \alpha\right)^{2}-\frac{1}{3} k^{2}=0 .
\end{aligned}
$$

The existence set $\Delta^{-1}(0)$ is a hyperbola, the $\mathscr{B}_{k}=0$ curve is a circle, and the $\mathscr{B}_{k k}=0$ curve consists of two straight lines. Where the three surfaces intersect correspond to points where the $\mathrm{mKP}-\mathrm{KD}^{+}$equation arises, occurring when the hyperbola is tangent to the circle, with an example of this given in figure 2. The intersection occurs when

$$
\omega=-\frac{9}{32} \alpha^{2}, \quad u:=\left|\Psi_{0}\right|^{2}=\frac{3}{8} \alpha, \quad k^{2}=\frac{3}{64} \alpha^{2},
$$

with $\alpha$ any positive number. Note that there are two distinct $k$ values due to the symmetry with respect to $k$ in $(4.4)$.

This calculation confirms that there exist parameter values where the mKP can potentially emerge. Now compute the values of the cofficients in (1.11), evaluated at the singular point (4.8)

$$
\mathscr{A}_{k}=-4 \frac{k}{\alpha}, \quad \mathscr{B}_{k k k}=-\frac{96}{\alpha}, \quad \mathscr{B}_{k m}=0, \quad \mathscr{C}_{m}=\left|\Psi_{0}\right|^{2}=\frac{3}{8} \alpha=\frac{8}{\alpha} k^{2} .
$$



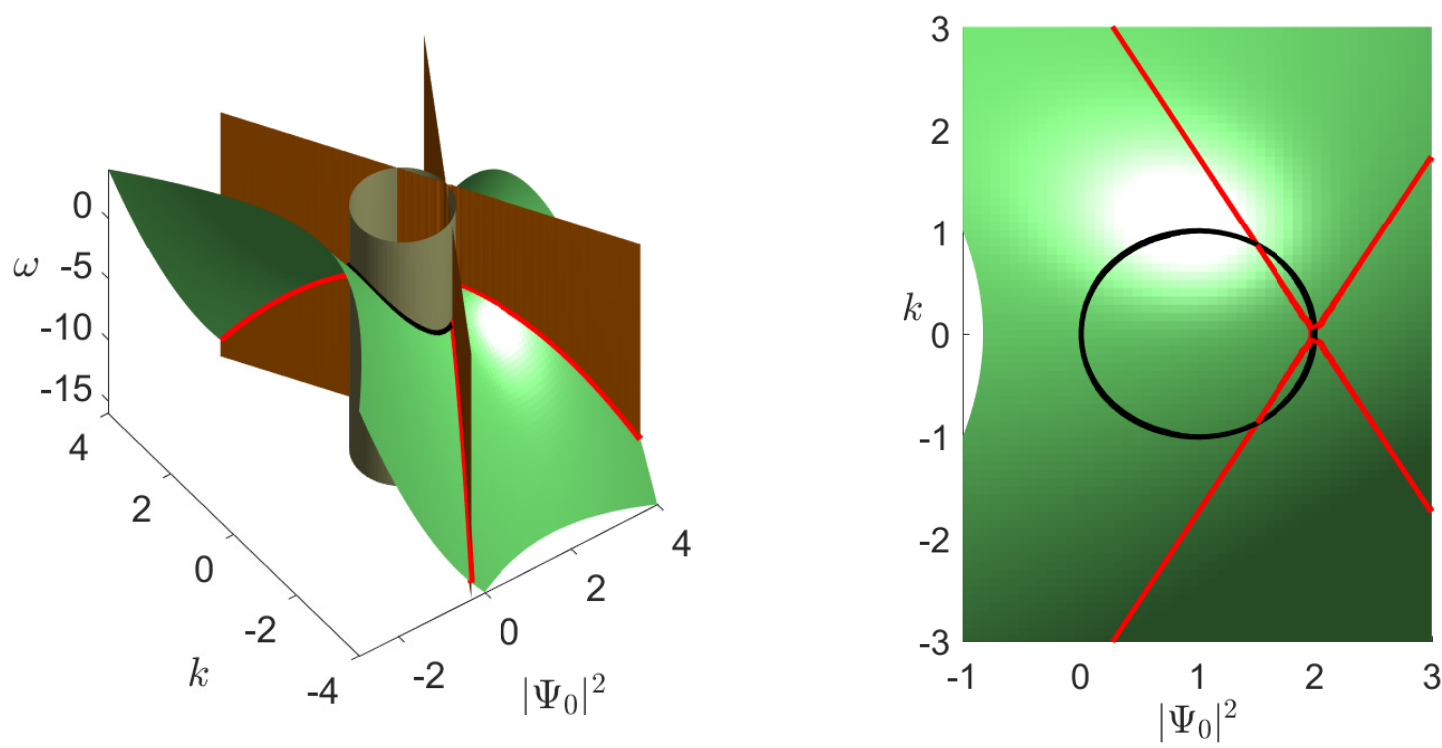

Figure 2: An example of the surfaces defined in (4.7) for $\alpha=4$. The surfaces corresponding to these conditions are coloured green, grey and orange respectively. The intersections between and the second and third conditions with the first are highlighted by the black and red lines respectively. The second image shows the intersection between these lines from above and thus the points where the mKP-KD ${ }^{+}$equation emerges.

In summary, just knowing that CQNLS comes from a Lagrangian and has a family of periodic travelling waves, with the symmetry in $m$ and the singularities $\mathscr{B}_{k}=0$ and $\mathscr{B}_{k k}=0$, it is immediate that there are parameter values where the mKP equation emerges in the form

$$
\left(-8 \frac{k}{\alpha} q_{T}-\frac{48}{\alpha} q^{2} q_{X}+\mathscr{K} q_{X X X}\right)_{X}+\frac{8}{\alpha} k^{2} q_{Y Y}=0 .
$$

It remains to compute the $X$-dispersion coefficient $\mathscr{K}$. This coefficient can be obtained by using the dispersion relation of (4.2) linearised about the family of basic states, or it can be calculated using the Jordan chain argument given in $\S 2.2$. The calculation is given below using the Jordan chain argument, and it is found that

$$
\mathscr{K}=-\frac{2}{\alpha}
$$

Substitution into (4.10) and simplifying gives the final form for the mKP emerging from CQNLS at the singular point (4.8),

$$
\left(k q_{T}+6 q^{2} q_{X}+\frac{1}{4} q_{X X X}\right)_{X}-k^{2} q_{Y Y}=0,
$$


noting that $k= \pm \alpha \sqrt{3} / 8$. By scaling it can be put in the canonical form (1.16). The resulting mKP equation is focussing and so the periodic travelling waves can be BenjaminFeir unstable [7].

\subsection{The dispersion coefficient $\mathscr{K}$}

To confirm the expression (4.11) for the dispersion coefficient the Jordan chain argument in $\S 2.2$ is used. Since $\mathscr{K}$ is associated with $X$-dispersion, it can be calculated using the 1D version of CQNLS,

$$
i \Psi_{t}+\Psi_{x x}-\alpha|\Psi|^{2} \Psi+|\Psi|^{4} \Psi=0 .
$$

First set up the multisymplectic structure $\mathbf{M}, \mathbf{J}$ and $S$, and construct the linearisation about the family of basic states. Let $\Psi=a_{1}+i a_{2}$ and introduce $\mathbf{a}=\left(a_{1}, a_{2}\right)$ and $\mathbf{b}=\mathbf{a}_{x}$. Then the multisymplectic formulation of (4.13) is

$$
\mathbf{M} Z_{t}+\mathbf{K} Z_{x}=\nabla S(Z)
$$

where,

$$
Z=\left(\begin{array}{l}
\mathbf{a} \\
\mathbf{b}
\end{array}\right), \quad \mathbf{M}=\left(\begin{array}{cc}
-\boldsymbol{\sigma} & \mathbf{0} \\
\mathbf{0} & \mathbf{0}
\end{array}\right), \quad \mathbf{J}=\left(\begin{array}{cc}
\mathbf{0} & -\mathbf{I} \\
\mathbf{I} & \mathbf{0}
\end{array}\right), \quad \boldsymbol{\sigma}=\left(\begin{array}{cc}
0 & -1 \\
1 & 0
\end{array}\right)
$$

and

$$
S(Z)=\frac{1}{2} \mathbf{b} \cdot \mathbf{b}-\frac{\alpha}{4}(\mathbf{a} \cdot \mathbf{a})^{2}+\frac{1}{6}(\mathbf{a} \cdot \mathbf{a})^{3} .
$$

Just a summary of the calculations are given since analysis of this type appears in previous papers $[1,16,17]$. The basic state in the multisymplectic representation is

$$
\widehat{Z}(\theta, k, m, \omega)=\mathrm{G}_{\theta}\left(\begin{array}{l}
\widehat{\mathbf{a}} \\
\widehat{\mathbf{b}}
\end{array}\right),
$$

with $|\widehat{\mathbf{a}}|^{4}-\alpha|\widehat{\mathbf{a}}|^{2}-\omega-k^{2}=0, \widehat{\mathbf{b}}=k \boldsymbol{\sigma} \widehat{\mathbf{a}}$, and

$$
\mathrm{G}_{\theta}:=\mathrm{R}_{\theta} \oplus \mathrm{R}_{\theta}, \quad \mathrm{R}_{\theta}:=\left[\begin{array}{cc}
\cos \theta & -\sin \theta \\
\sin \theta & \cos \theta
\end{array}\right]
$$

Linearising (4.14) about the family of basic states generates the linear operator

$$
\mathbf{L}:=\mathrm{D}^{2} S\left(\widehat{Z}_{\theta}\right)-k \mathbf{J} \partial_{\theta}-\omega \mathbf{M} \partial_{\theta}
$$

or, after substitution,

$$
\mathbf{L}:=\left[\begin{array}{cc}
\left(4|\mathbf{a}|^{2}-2 \alpha\right) \mathbf{a a}^{T}+k^{2} \mathbf{I} & k \boldsymbol{\sigma} \\
-k \boldsymbol{\sigma} & \mathbf{I}
\end{array}\right]
$$


The kernel of $\mathbf{L}$ is spanned by

$$
\xi_{1}:=\widehat{Z}_{\theta}=\mathrm{G}_{\theta}\left(\begin{array}{c}
\boldsymbol{\sigma} \widehat{\mathbf{a}} \\
-k \widehat{\mathbf{a}}
\end{array}\right) .
$$

The next two members of the Jordan chain satisfy $\mathbf{L} \xi_{2}=\mathbf{J} \xi_{1}$ and $\mathbf{L} \xi_{3}=\mathbf{J} \xi_{2}$. These equations are easily solved explicitly giving

$$
\xi_{2}=\mathrm{G}_{\theta}\left(\begin{array}{c}
\beta \widehat{\mathbf{a}} \\
(1+k \beta) \boldsymbol{\sigma} \widehat{\mathbf{a}}
\end{array}\right) \quad \text { and } \quad \xi_{3}=\mathrm{G}_{\theta}\left(\begin{array}{c}
0 \\
\beta \widehat{\mathbf{a}}
\end{array}\right),
$$

with

$$
\beta:=\frac{k}{\left(2|\widehat{\mathbf{a}}|^{2}-\alpha\right)|\widehat{\mathbf{a}}|^{2}}
$$

Now using the formula (2.9),

$$
\mathscr{K}=\left\langle\left\langle\xi_{2}, \mathbf{J} \xi_{3}\right\rangle\right\rangle=-\beta^{2}|\widehat{\mathbf{a}}|^{2}=-\frac{2}{\alpha} .
$$

Confirming (4.11).

\section{Concluding remarks}

In this paper the $x$-direction was preferred in that it was the singularities $\mathscr{B}_{k}=\mathscr{B}_{k k}=0$ that underpinned the derivation. There is a dual version where time is the preferred direction. In the $1+1$ case the singularities (1.8) are replaced by $\mathscr{A}_{\omega}=\mathscr{A}_{\omega \omega}=0$ and the resulting modulation equation is

$$
\left(\mathscr{B}_{\omega}+\mathscr{A}_{k}\right) \Omega_{X}+\frac{1}{2} \mathscr{A}_{\omega \omega \omega} \Omega^{2} \Omega_{T}+\mathscr{M} \Omega_{T T T}=0
$$

with $\mathscr{M}$ determined by a $\mathbf{M}$-based Jordan chain. This equation has $2+1$ generalisations, and could also be oriented so that $y$ is the preferred direction.

Although related to the $\mathrm{KdV}$ equation, the $\mathrm{mKdV}$ equation has different structure and a wider range of solutions. For example the focusing $\mathrm{mKdV}$ equation has periodic travelling wave solutions which are Benjamin-Feir unstable [7]. It is shown in [3, 4] that this BenjaminFeir instability can be the catalyst for an energy cascade leading to a continuous spectrum and a highly-complex wave field.

When $\mathscr{B}_{k k}$ is not zero but small, for example $\mathscr{B}_{k k}=\varepsilon \mu$, the mKP-KD ${ }^{+}$has an unfolding into the $2+1$ Gardner system which includes both cubic and quadratic nonlinearities.

$$
\begin{aligned}
\left(\left(\mathscr{A}_{k}+\mathscr{B}_{\omega}\right) q_{T}+\mu q q_{X}+\frac{1}{2} \mathscr{B}_{k k k} q^{2} q_{X}+\mathscr{K} q_{X X X}\right)_{X} & \\
& +\mathscr{C}_{m} q_{Y Y}+\left(\mathscr{B}_{k m}+\mathscr{C}_{k k}\right)\left(q q_{Y}\right)_{X}+\mathscr{B}_{k m}\left(q_{X} \partial_{X}^{-1} q_{Y}\right)_{X}=0 .
\end{aligned}
$$


The most important potential generalisation of the theory in this paper is to the case where the symmetry has dimension greater than one, the case of multiphase modulation. The case of multiphase modulation in the case of a codimension one singularity leading to $\mathrm{KdV}$ is developed in [18]. The generalisation of this theory to the codimension two case is of great interest since the most well-known example of the emergence of the mKdV equation is in multi-layer stratified shallow water hydrodynamics [6] and that case can be viewed as multiphase modulation with a multi-dimensional affine symmetry group. Some progress in this direction is in [15], where a theory for codimension two multiphase modulation leading the Boussinesq equation (a multiphase generalisation of the codimension two singularity in the left of Figure 1) is presented.

\section{Acknowledgements}

The first author is funded by an EPSRC PhD studentship with grant number EP/L505092/1.

[1] Bridges T 2013 A universal form for the emergence of the Korteweg-de Vries equation, Proc. Roy. Soc. Lond. A 46920120707.

[2] Bridges T \& FurTer J 1993 Singularity Theory and Equivariant Symplectic Maps, Lect. Notes Math. 1558, (Berlin: Springer-Verlag).

[3] Dutykn D and Tobisch E 2015 Observation of the inverse energy cascade in the modified Kortewegde Vries equation, Euro. Phys. Lett. 10714001.

[4] Dutykh D and Tobisch E 2015 Direct dynamical energy cascade in the modified KdV equation, Physica D 297 76-87.

[5] Golubitsky M, Stewart I, \& Schaeffer D 1988 Singularities and Groups in Bifurcation Theory, Volume II, (New York: Springer-Verlag).

[6] Grimshaw R, Pelinovsky E, Talipova T 1997 The modified Korteweg-de Vries equation in the theory of large-amplitude internal waves, Nonl. Proc. Geophys. 4 237-250.

[7] Grimshaw R, Pelinovsky D, Pelinovksy E and Talipova, T 2001 Wave group dynamics in weakly nonlinear long-wave models, Physica D 159 35-57.

[8] Herrmann J 1992 Bistable bright solitons in dispersive media with a linear and quadratic intensitydependent refraction index change, Opt. Commun. 87, 161-165.

[9] Kevrekidis P, Frantzeskakis D, \& Carretero-González R 2015 The Defocusing Nonlinear Schrödinger Equation, (Philadelpha: SIAM).

[10] Konopelchenko B On the gauge-invariant description of the evolution equations integrable by Gelfand-Dikij spectral problems, Phys. Lett. A 92 323-327.

[11] Konopelchenko B and Dubrovsky V 1984 Some new integrable nonlinear evolution equations in 2+1 dimensions, Phys. Lett. A 102, 15-17.

[12] MiURA R 1968 Korteweg-de Vries equation and generalizations. I. A remarkable explicit nonlinear transformation, J. Math. Phys. 91202.

[13] Muryshev A, Shlyapnikov G, Ertmer W, Sengstock K, \& Lewenstein M 2002 Dynamics of dark soltons in elongated Bose-Einstein condensates, Phys. Rev. Lett. 89110401.

[14] RatlifF D 2017 Phase dynamics of periodic wavetrains leading to the fifth-order KP equation, Physica $D$ in press: https://doi.org/10.1016/j.physd.2017.05.004

[15] RatLifF D 2017 Double degeneracy in multiphase modulation and the emergence of the Boussinesq equation, Preprint, submitted. 
[16] Ratliff D And Bridges T 2015 Phase dynamics of periodic waves leading to the KadomtsevPetviashvili equation in 3+1 dimensions, Proc. R. Soc. Lond. A 47120150137.

[17] Ratliff D AND BRidges T 2016 Whitham modulation equations, coalescing characteristics, and dispersive Boussinesq dynamics, Physica D 333 107-116.

[18] Ratliff D AND BRIDGes T 2016 Multiphase wavetrains, singular wave interactions, and the emergence of the KdV equation, Proc. R. Soc. Lond. A 47220160456.

[19] Whitham G 1974 Linear and Nonlinear Waves, (New York: John Wiley \& Sons).

[20] Zhang D-J, Zhao S-L, Sun Y-Y, Zhou J 2014 Solutions to the modified Korteweg-de Vries equation, Rev. Math. Phys. 261430006. 